\title{
Review on Biosynthesis, Characterization and Antibacterial Activity of Silver Nanoparticles
}

\author{
Tizeta Abera \\ Department of Chemistry, Arba Minch University, Arba Minch, Ethiopia
}

Email address:

tizetaabera27@gmail.com, Tizeta.abera@amu.edu.et

\section{To cite this article:}

Tizeta Abera. Review on Biosynthesis, Characterization and Antibacterial Activity of Silver Nanoparticles. International Journal of Materials Science and Applications. Vol. 9, No. 3, 2020, pp. 47-52. doi: 10.11648/j.ijmsa.20200903.12

Received: June 23, 2020; Accepted: August 8, 2020; Published: October 12, 2020

\begin{abstract}
Recently, nanotechnology has attracted great attention due to its wide applications for different fields of science. Nanoparticles are a cluster of atoms in the range of 1-100 nm that provides mechanical, optical, electrical, and structural advanced, and also an increased surface area than the original substance. Nanostructure materials have got an enhancement to the feature of life and preservation of the environment. Now a day's silver nanoparticles have gained attention due to its uses in various areas of human interest in the industry, medicine, human health, and agriculture. It is the most popular metallic nanoparticles in antimicrobial, antioxidant, and anti-cancer properties as different researchers reported. The objective of this review is to brief recent progress in the biosynthesis of AgNPs. This review may have a great contribution in the field of green synthesis, characterization, and antibacterial activities of AgNPs. There are three types of nanoparticles synthesis approaches they are physical, chemical, and biological methods. The biological method for the synthesis of AgNPs is a preferable approach due to its simplicity, cost-effectiveness, easily scale up to the industry and non-toxicity. Plants and their parts contain carbohydrates, fats, proteins, nucleic acids, pigments, and several types of secondary metabolites which act as reducing agents to produce nanoparticles from metal salts without producing any toxic by-product. In this review, different researches reported different particle size of AgNPs $(0.011-90 \mathrm{~nm})$ and shape by using different plant material. I conclude This review is concerned with the green synthesis of AgNPs, characterization, and antibacterial activity of AgNPs.
\end{abstract}

Keywords: Antibacterial Activity, Characterization, Green Synthesis, Nanoparticles, Silver Nanoparticles

\section{Introduction}

\subsection{Nanotechnology}

Nanotechnology study about the synthesis of nanoparticles with controlled size, shape purity, and disparity of materials at the nanometer scale length and their application in different fields. It is an emerging industry that is bringing us exciting new products and promises to change the way we live and work in the future. This field of science is developing from time to time and is making a valuable impact on recent science. It is applicable in different fields such as health care, food science, cosmetics, energy science, electronics, mechanics, space industries, environmental health, biomedical science, chemical industries, drug, and gene delivery It also has expansively been achieved for the treatments of cancer, diabetes, allergy, infection, and inflammation [1-3]

\subsection{Nanoparticles}

Nanoparticles are a cluster of atoms in the range of 1-100 $\mathrm{nm}$ that provides mechanical, optical, electrical, and structural advanced, and an increased surface area than the original substance. As particle size decreases, the surface area-to-volume ratio of nanoparticles increases radically it leads to significant changes in their physical, chemical, and biological properties $[1,4,5]$. Nanoparticles are now considered as a feasible alternative, to antibiotics and show to have a high potential to solve the problem on the emergence of bacterial multidrug resistance.

\subsection{Synthesis of Nanoparticles}

There are different types of processes for the synthesis of nanoparticles it can be classified into three types, those are physical synthesis, chemical synthesis, and biological synthesis 
[4] however, the physical and chemical routes of nano-particles preparation have many disadvantages and are not eco-friendly. Therefore, researchers across the globe have searched for new and environmentally friendly methods for the synthesis of biocompatible nanoparticles. Biosynthesis (green synthesis) of nano-silver has received extensive attention due to the growing need for environmentally friendly synthesis methods that use biological reducing and capping agents, such as various species of bacteria, fungi, yeast, algae, and plants [4, 6].

The physical and chemical routes of nanoparticle preparation have many disadvantages and are not eco-friendly. Hence, researchers across the globe have searched for new and environmentally compatible methods for the synthesis of nanoparticles.

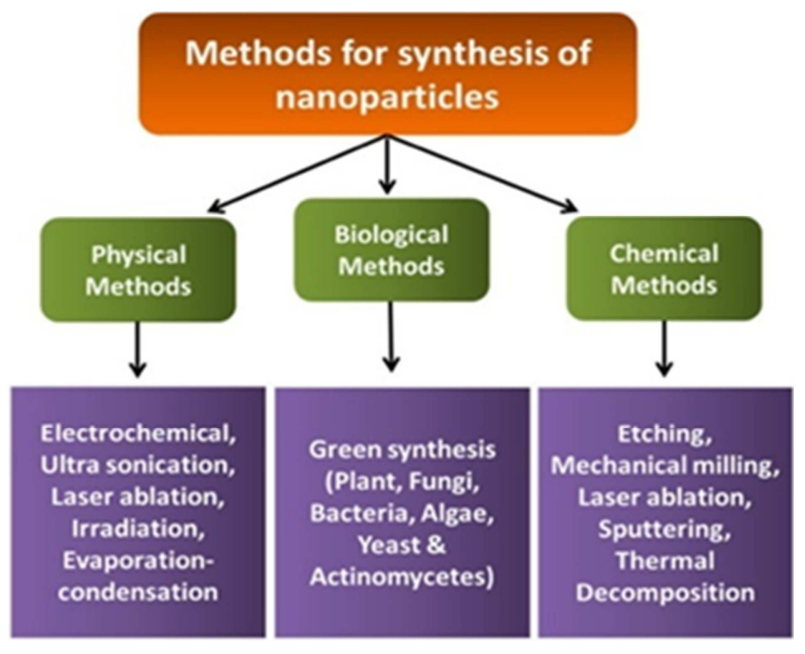

Figure 1. Different methods for synthesis of nanoparticles.

\subsection{Green Synthesis of Metal Nanoparticles}

Unlike physical and chemical processing, biological synthesis is further preferable in which the biological system provides an innovative research idea in nanoscale materials production. Numerous researchers suggested that using a biological synthesis of AgNPs, without using expensive and hazardous chemical agents such as organic solvents and the toxic reducing agent is important to enhance environmentally friendly technologies. Consequently, biosynthesis strategies are very simple techniques and cost-effective, short time required, and can easily be scaled up for industrial purposes [6].

Biosynthesis of nanoparticles is an approach that is compatible with green chemistry approaches in which the bio-molecules can act as both reducing and stabilizing agents during the reaction. In recent years, plant-based synthesis of nanoparticles gained much attention due to its rapidity and its simplicity. In nanoparticles synthesis using plant extracts, the extract of the plant is simply mixed with the aqueous solution of the metal salt at room temperature, so that these processes can be considered as a green synthesis approach. The actual mechanism for the synthesis of nanoparticles remains the same for microorganisms and plants. Metal salts consisting of metal ions are first reduced using reducing agents. The obtained atoms then aggregated and form small clusters that grow into particles [6-8].

\subsection{Biosynthesis of Silver Nanoparticles by Using Plant}

Plant extracts have enzymes and phytochemicals such as terpenoids, flavonoids and phenolic compound. Which act as bio-reductants as well as capping agent in the presence of metal salt for nanoparticles synthesis. The major advantage of biological methods is the availability of amino acids, proteins, or secondary metabolites present in the synthesis process, the elimination of the extra step required for the prevention of particle aggregation, and the use of biological molecules for the synthesis of AgNPs is eco-friendly and pollution-free. Biological methods seem to provide controlled particle size and shape, which is an important factor for various biomedical applications $[7,9]$.

\subsubsection{Preparation of Parts of Plant Extract}

Different researches reported different technique for preparation of plant extract. The most common preparation method is first collecting the part of plant that may be leaf, seed, flower, and tuber etc. secondly wash with running water to remove dirty particle then rinse with distilled and deionized water to remove debris and other contaminated organic contents. After that it will dry at room temperature and grind by using mortar and pestel. The powder will be then stored in well labeled airtight container.

Aqueous plant extract can be prepared by mixing $10 \mathrm{~g}$ of powder with $100 \mathrm{~mL}$ distilled water in $1000 \mathrm{~mL}$ of Erlenmeyer flask and boiled for 30 minutes at $60^{\circ} \mathrm{C}$, then cooled down at room temperature and the supernatant will be separated and filtered through Whatman No.1 filter paper to remove particulate matter and to get aqueous extract of plant. The filtrate will collected and stored at $4{ }^{\circ} \mathrm{C}$ for further use as a reducing, capping and stabilizing agent for the synthesis of AgNPs without further treatment.

\subsubsection{Synthesis of Ag NPs. with Plant}

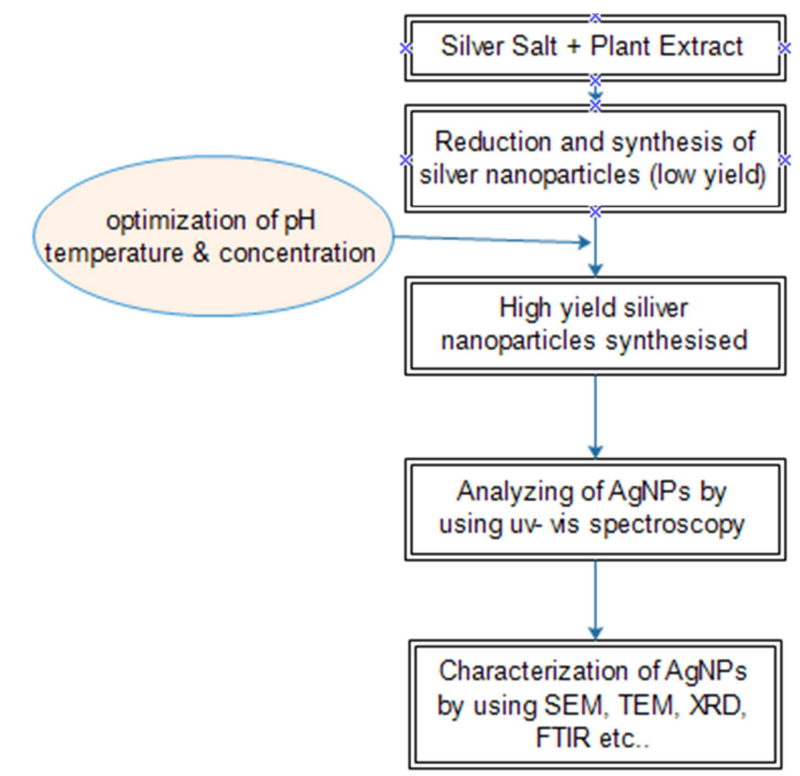

Figure 2. Biosynthesis of AgNPs and their optimization techniques. 
Aqueous solution $0.001 \mathrm{M}\left(\mathrm{AgNO}_{3}\right)$ will be prepared and use for the synthesis of silver nanoparticles. $3 \mathrm{ml}$ plant extract will be mixed with $40 \mathrm{ml}$ of $0.001 \mathrm{M} \mathrm{AgNO}_{3}$ solution The synthesis of silver nanoparticles carried out at room temperature for $24 \mathrm{~h}$ in dark.

The synthesized AgNPs will be optimized by using different parameters volume of silver Nitrate and volume of plant aqueous extract, heating temperature and heating time of the plant extract and $\mathrm{pH}$ value.

\section{Characterization of Nanoparticles}

The importance of the characterization of nanoparticles is to investigate the physical and chemical behavior of nanoparticles. Therefore, the importance of the characterization of AgNPs is to evaluate its size, shape, morphology, crystalline nature, optical properties, and the purities of synthesized particles. Two of the main parameters studied in the characterization of NPs are size and shape. By using different analytical techniques, like UV-vis spectroscopy, $\mathrm{X}$-ray diffractometry (XRD), Fourier transform infrared spectroscopy (FTIR), scanning electron microscopy (SEM), and transmission electron microscopy (TEM), the characterization is performed $[9,10]$.

\subsection{Uv-vis Spectroscopy}

UV-vis spectroscopy is a useful and reliable technique for the primary characterization of synthesized nanoparticles. It examines the synthesis and stability of AgNPs. It works by measuring the intensity of light reflected from a sample and compares it to the intensity of light reflected from reference material. Nanoparticles have optical properties that are sensitive to size, shape, concentration, agglomeration, and refractive index near to the surface of the nanoparticles. It makes UV-Vis spectroscopy an Important tool to identify and evaluate the stability of NP colloidal solutions. Silver nanoparticles have unique optical properties. It can strongly interact with specific wavelengths of light. Besides, UV-vis spectroscopy is a fast, easy, simple, low cost, sensitive, and selective for different types of NPs $[9,10]$.

\subsection{FTIR}

Fourier Transform Infrared [FTIR] spectroscopy Measures infrared intensity vs. wavelength of light, it is used to determine the nature of associated functional groups and structural features of biological extracts with nanoparticles. The calculated spectra reflect the well-known dependence of nanoparticle optical properties. The green synthesized silver nanoparticles by employing various plant extract was analyzed using Fourier Transform Infrared [FTIR] Spectroscopy showed characteristic peaks. Furthermore, FTIR has also been extended to the study of nano-scaled materials, such as confirmation of functional molecules covalently grafted onto silver, carbon nanotubes, graphene, and gold nanoparticles, or interactions occurring between enzyme and substrate during the catalytic process $[9,10]$.

\subsection{XRD}

X-ray diffraction (XRD) is a popular analytical technique that has been used for the analysis of both molecular and crystal structures. XRD is a primary technique for the identification of crystalline nature at the atomic scale. It is a nondestructive technique with great potential for the characterization of both organic and inorganic crystalline materials. This method has been used to measure phase identification, conduct quantitative analysis, and to determine structure imperfections in samples from various disciplines, such as geological, polymer, environmental, pharmaceutical, and forensic sciences. Recently, the applications have extended to the characterization of various nanomaterials and their properties $[9,10]$.

\subsection{SEM}

Recently, the field of nanoscience and nanotechnology has provided a driving force in the development of various high-resolution microscopy techniques to learn more about nanomaterials using a beam of highly energetic electrons to probe objects on a very fine scale. Among various electron microscopy techniques, SEM is a surface imaging method, fully capable of resolving different particle sizes, size distributions, nanomaterial shapes, and the surface morphology of the synthesized particles at the micro and nanoscales. The characterization of Scanning electron microscope analysis is employed to determine the size, shape \& morphologies of formed nanoparticle SEM gives high-resolution images of the surface of a sample is desired. The scanning electron microscope works as the same principle as an optical microscope, but it measures the electrons scattered from the sample rather than a photon. Because electrons can be accelerated by an electric potential, the wavelength can be made shorter than the one of photons. This makes the SEM capable of magnifying images up to 200.000 times $[9,10]$.

\subsection{TEM}

TEM is a valuable, frequently used, and important technique for the characterization of nanoparticles used to obtain quantitative measures of particle size and morphology. It is a microscopy technique in which a beam of electrons is transmitted through an ultra-thin specimen, interacting with the specimen as it passes through. An image is formed from the interaction of the electrons transmitted through the specimen; the image is magnified and focused onto an imaging device, such as a fluorescent screen, on a layer of photographic film, or to be detected by a sensor such as a CCD camera $[9,10]$. The magnification of TEM is mainly determined by the ratio of the distance between the objective lens and the specimen and the distance between the objective lens and its image plan. TEM has two advantages over SEM: it can provide better spatial resolution and the capability for additional analytical measurements. The disadvantages include a required high vacuum, thin sample section, and the vital aspect of TEM is that sample preparation is 
time-consuming. Therefore, sample preparation is extremely important to obtain the highest-quality images possible [9].

\section{Antibacterial Activity of Silver Nanoparticles}

Since ancient times among various antimicrobial agents, Ag has been most extensively studied and used to fight against infections and prevent spoilage. AgNPs are among the most widely commercialized engineered nano-materials, because of their antimicrobial properties. Silver has greater toxicity against a wide range of microorganisms. AgNPs are found to have anti-inflammatory, anti-angiogenesis, antiviral, anti-platelet, and anti-cancer properties, which makes them a very potent agent in various fields of biology, chemistry, and medicine. It is commonly used in medical devices, household products, and industry. AgNPs are non-toxic to animal cells and highly toxic to bacteria, and other microorganisms (E-coli, Pseudomonas aeruginosa, Staphylococcus aureus). Due to these phenomena, it is considered to be safe and effective bactericidal metal $[1,2,5,11]$.

AgNps is known for its antimicrobial properties and has been used for years in the medical field for antimicrobial applications and even has shown to prevent HIV binding to host cells. The AgNPs are also reported to be non-toxic to humans and effective against bacteria, viruses, and other eukaryotic micro-organisms at very low concentrations and without any side effects. AgNPs, because of their large specific surface area, are highly active and can play a crucial role in inhibiting bacterial growth in aqueous and solid media. The antimicrobial activity of colloidal $\mathrm{Ag}$ is influenced by the size of the particles. The smaller the particle size has a high antimicrobial effect $[8,11]$.

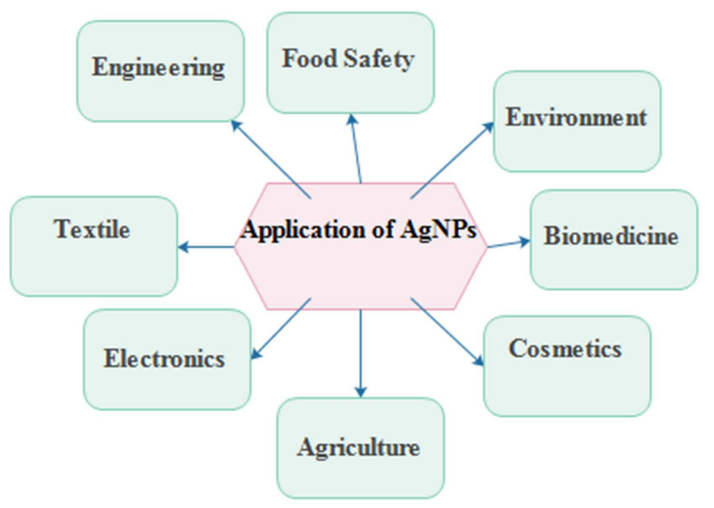

Figure 3. Application of AgNPs for Difference field of science.

Table 1. Nanoparticles synthesized by different plants and their particle size and shape.

\begin{tabular}{|c|c|c|c|c|c|}
\hline No & Types of plant & Part of plant & Particle size & Particle shape & Ref. \\
\hline 1 & Dioscorea bulbifera & Tuber & $13.54 \mathrm{~nm}$ & Nanorods and triangles & [12] \\
\hline 2 & Aloe Vera & Leaf & $68.7-70 \mathrm{~nm}$ & rectangular, triangular and spherical & [13] \\
\hline 3 & Fenugreek & Seed & $17 \mathrm{~nm}$ & Spherical & [14] \\
\hline 4 & Pimpinella anisum & Seed & $3.2-16 \mathrm{~nm}$ & Spherical & {$[15]$} \\
\hline 5 & Jatropha curcas & Seed & $15-50 \mathrm{~nm}$ & Spherical & [16] \\
\hline 6 & Nigella Sativa & Seed & $77.7 \mathrm{~nm}$ & Nanorods & [17] \\
\hline 7 & Cuminum cyminum & Seed & $13 \mathrm{~nm}$ & Not explained & {$[18]$} \\
\hline 8 & Phoenix dactylifera & Seed & $14-30 \mathrm{~nm}$ & Spherical & [19] \\
\hline 9 & Alpinia katsumadai & Seed & $12.6 \mathrm{~nm}$ & Spherical & {$[20]$} \\
\hline 10 & Pisum sativum & Seed & $3-36 \mathrm{~nm}$ & Spherical & {$[21]$} \\
\hline 11 & Matricaria chamomilla & Flowers & $5-30 \mathrm{~nm}$ & Spherical & {$[22]$} \\
\hline 12 & Calendula officinalis & Flowers & $5-30 \mathrm{~nm}$ & Spherical & [22] \\
\hline 13 & Lavandula angustifolia & Flowers & $5-30 \mathrm{~nm}$ & Spherical & [22] \\
\hline 14 & Eriobotrya japonica & Leaf & $9.26-11.98 \mathrm{~nm}$ & Not clearly predicted & [23] \\
\hline 15 & Andrographis Paniculata & Leaf & $24-90 \mathrm{~nm}$ & Not clearly predicted & [24] \\
\hline 16 & Azadirachta indica & Leaf & $34 \mathrm{~nm}$ & Spherical & {$[25]$} \\
\hline 17 & Olive & Leaf & $20-25 \mathrm{~nm}$ & Spherical & [26] \\
\hline 18 & Tarragon & Leaf & $25.12 \mathrm{~nm}$ & quasi-spherical & [27] \\
\hline 19 & Araucaria Heterophylla & Leaf & $70-90 \mathrm{~nm}$ & Spherical & [28] \\
\hline 20 & Cestrum nocturnum & Leaf & $20 \mathrm{~nm}$ & Spherical & [29] \\
\hline 21 & Annona reticulata & Leaf & $5.28-8.31 \mathrm{~nm}$ & Spherical & [30] \\
\hline 22 & Psidium guajavaL. & Leaf & $0.011-0.020 \mathrm{~nm}$ & Not clearly predicted & [31] \\
\hline 23 & Tectona grandis & Seed & $10-30 \mathrm{~nm}$ & oval, spherical & [32] \\
\hline 24 & Tilia cordata, & Flowers & $50 \mathrm{~nm}$ & Spherical & [33] \\
\hline
\end{tabular}

\section{Conclusion}

Nowadays green chemistry and the use of green synthesis of nanoparticles lead a desire to develop environment-friendly techniques. Biosynthesis of silver nanoparticles using plant extracts has a great advantage as it is a low cost, environmentally compatible, it can be prepared quickly, has a simple procedure, and can scale up to the industry. In this review, I have tried to show the results of silver nanoparticles synthesized by different plant materials used as stabilizing and reducing agent. As a result show, the different plant material has different nanoparticles sizes and shapes. Green synthesized silver nanoparticles have significant aspects of nanotechnology through wide applications and synthesis of nanoparticles using plants is beneficial than the other nanoparticles synthesis method. Thus the present review showed comprehensively addressed synthesis, characterization, and antibacterial activity of silver nanoparticles. 


\section{References}

[1] Salomoni, R.; Léo, P. and Rodrigues, M. (2015) Antibacterial Activity of Silver Nanoparticles (AgNPs) in Staphylococcus aureus and Cytotoxicity Effect in Mammalian Cells. Battle Again. Micro. Patho.: Basic Science, Techno. Advances \& Edu. Prog, 851-857.

[2] Kumar, R. and Chouhan, N. (2015) Biosynthesis of Silver Nanoparticles from Plant (Fenugreek Seeds) Reducing Method and their Optical Properties. Res. J. Recent. Sci., 4, 47-52.

[3] Chandran, K. and, Indira G. Green synthesis, characterization and antimicrobial activity of silver nanoparticles using Morindapubscens J. E. Smith root extract. J. Sci. Innov. Res., 5 (3), 2016, 83-86.

[4] Ge, L.; Li, Q., wang, M.; Ouyang, J.; Li, X. and Xing, M. (2014) Nanosilver particles in medical applications: synthesis, performance, and toxicity. Int. J. of Nanomed., 9, 2399-2407.

[5] Varghese, R.; Mohammed, A.; Ilavenil, S. Rebecca, J. and Choon, k. (2019) Silver nanoparticles synthesized using the seed extract of Trigonella foenum-graecum L. and their antimicrobial mechanism and anticancer properties. Saudi $j$. boil. sci., 26, 148-154.

[6] Ravindra, B.; Kishor, S. and Machindra, J. (2018) Emerging Use of Green Synthesis Silver Nanoparticle: An Updated Review. Inter. J. Pharm. Sci. \& Res., 9, 4029-4055.

[7] Khandel, P.; Kumar, R.; Deepak, Y.; Soni, K.;'Kanwar, L. and Kumar, S. (2018) Biogenesis of metal nanoparticles and their pharmacological applications: present status and application prospects. J. Nanostructure in Chem., 1-39.

[8] Luis, J.; Justin, L.; Jose, R, Camacho, A.; Gao, X.; Humberto, H. and Jose, M. (2005) Interaction of silver nanoparticles with HIV-1. J. Nanobiotechn., 6, 1-10.

[9] Zhang, X.; Liu, Z.; Shen, W. and Gurunathan, S. (2016) Silver Nanoparticles: Synthesis, Characterization, Properties, Applications, and Therapeutic Approaches. Int. J. Mol. Sci. 17, 1534.

[10] Heera, P. and Shanmugam, S. (2015) Nanoparticle Characterization and Application. Int. J. Curr. Microbiol. App. Sci. 4: 379-386.

[11] Hailemariam, G.; Abi, T.; Jyotsna, K. And Yadav, O. (2013) Green Synthesis of Silver Nanoparticles and their Antibacterial Activity. J. Surface Sci. Technol., 29, 47-66.

[12] Ghosh, S.; Patil, S.; Ahire, M.; Kitture, R.; Kale, S.; Pardesi, K cameotra, S.; Bellare, J.; Dhavale, D.; Jabgunde, A. and chopade, B. (2012) synthesis of silver nanoparticles using Dioscorea bulbifera tuber extract and evaluation of its synergistic potential in combination with antimicrobial agents. Int. J. Nanomedicine. 7, 483-496.

[13] Shreya, M.; Amita, H.; Uttiya, D.; Paulomi, B. and Naba, K. (2014) Biosynthesis of silver nanoparticles from aloe vera leaf extract and antifungal activity against Rhizopussp. And Aspergillussp. Appl Nanosci 5: 875-880.

[14] Nabila, H.; Shaarawy, H.; Hawas, S. and Amal, E. (2018) Green Synthesis Of Silver Nano Particles Using Fenugreek Seeds Extract. ARPN Journal of Engineering and Applied Sciences. 13, 417-422.
[15] Alsalhi, M.; Devanesan, S.; Alfuraydi, A.; Vishnubalaji, R.; Munusamy, M.; Murugan, K.; Nicoletti, M. and Benelli, G. Green synthesis of silver nanoparticles using Pimpinella anisum seeds: antimicrobial activity and cytotoxicity on human neonatal skin stromal cells and colon cancer cells Int. J. Nanomedicine. 11, 4439-4449.

[16] Bar, H.; Bhui, D.; Gobinda, P.; Sarkar, P.; Santanu Pyne and Ajay Misra (2009) Green synthesis of silver nanoparticles using seed extract of Jatropha curcas. Colloids and Surfaces A: Physicochem. Eng. Aspects 339, 134-139.

[17] Mukesh, P.; Vinmathi, V.; Gautam, P.; Herald, A. and Justin, S. (2015) Green Synthesis of Silver Nanorods using Aqueous Seed Extract of Nigella Sativa and Study of its Antidiabetic Activity. Australian Journal of Basic and Applied Sciences. 9, 295-298.

[18] Jeyashree and Revathi. (2017) Synthesis of AgNPs of Cuminum cyminum seed extract, Characterization, and Antimicrobial Activity. International Journal of Engineering Science and Computing, 7, 1419-14196.

[19] Mohammad, A. and Mohammad, A. (2018) One-Pot Facile Green Synthesis of Silver Nanoparticles Using Seed Extract of Phoenix dactylifera and Their Bactericidal Potential against MRSA Evidence-Based Complementary and Alternative Medicine.

[20] Yangqing, H.; Fenfei, W.; Zhanying, M.; Hao, Z.; Qian, Y., Binghua, Y.; Zhengrui, H.; Jie, L.; Cun, Z. and Qian, Z. (2017) Green synthesis of silver nanoparticles using seed extract of Alpinia katsumadai, and their antioxidant, cytotoxicity, and antibacterial activities. The Royal Society of Chem. 7, 39842-39851.

[21] Nilesh, S. and Raman, P. (2015) Biosynthesis of silver nanoparticles using plant seeds and their antimicrobial activity Asian Journal of Biomedical and Pharmaceutical Sciences, 5 (45), 26-28

[22] Mladenova, B.; Diankov, S.; Karsheva, M.; Stankov, S. and Hinkov, I. (2018) Plant Mediated Synthesis Of Silver Nanoparticles Using Extracts From Tilia Cordata, Matricaria Chamomilla, Calendula Officinalis, And Lavandula Angustifolia Flowers. Journal of Chemical Technology and Metallurgy. 53, 623-630.

[23] Yu, C.; Tang, J.; Liu, X., Ren, X.; Zhen, M and Wang, L. (2018) Green Biosynthesis of Silver Nanoparticles Using Eriobotrya japonica (Thunb.) Leaf Extract for Reductive Catalysis. Materials, 12, 189.

[24] Saratha, V.; Subasri, S. And Usharani, S. (2018) Synthesis of Silver Nanoparticles From Andrographis Paniculata and Evaluation of Their Antibacterial Activity. Innovare Journal of Life Sciences. 6, 10-14.

[25] Ahmed, S.; Saifullah; Ahmad, M.; Swami, B. and Ikram, S. (2016) Green synthesis of silver nanoparticles using Azadirachta indica aqueous leaf extract. Journal of Radiation Research and Applied Sciences, 9, 1-7.

[26] Mostafa, M.; Eman, H.; Khaled, Z. and Mohamed, D. (2014) Green synthesis of silver nanoparticles using olive leaf extract and its antibacterial activity. Arabian Journal of Chemistry. 7 , 1131-1139.

[27] Omidi, S.; Sedaghat, S.; Tahvildari, K.; Derakhshi, P. and Motiee, F. (2018) Biosynthesis of silver nanocomposite with Tarragon leaf extract and assessment of antibacterial activity. $J$. Nanostru. in Chem., 8, 171-178. 
[28] Grace, H.; and Albin T. (2018). Biosynthesis of Silver Nanoparticles Using Leaf Extract of Araucaria Heterophylla And Its Activity Against Breast Cancer Cell Line. 7, 975-982.

[29] Kumar, A.: Srivastava, R.; Singh, P.; Bahadur, V. and Nath, G. (2018) Antioxidant and antibacterial activity of silver nanoparticles synthesized by Cestrum nocturnum. Journal of Ayurveda and Integrative Medicine, 1-8.

[30] Parthiban, E.; Manivannan, N.; Ramanibai, R. and Mathivanan N. (2019) Green synthesis of silver-nanoparticles from Annona reticulata leaves aqueous extract and its mosquito larvicidal and anti-microbial activity on human pathogens. Biotechnology Reports, 20, 1-10.

[31] Moteriya, P.; Padalia, H. and Chanda, S. (2014) Green biosynthesis of silver nanoparticles using Psidium guajavaL. leaf extract and antibacterial activity against some pathogenic microorganisms. J. Pharm. Rese., 11, 1579-1585.

[32] Rautela, A.; Rani, J. and Debnath, M. (2019) Green synthesis of silver nanoparticles from Tectona grandis seeds extract characterization and mechanism of antimicrobial action on different microorganisms. J. Anal. Sci. e \& Techn., 10, 1-10.

[33] Corciova, A.; Ivanescu, B.; Tuchilus, C.; Fifere, A.; Doroftei, F.; Lungoci, A.; Marangoci, N. and Mircea, C. (2018) Biosynthesis Of Silver Nanoparticles (AgNPs) Using Tilia Cordata Flowers Extracts And Evaluation Of Some Biological Activities. Environmental Engineering and Management Journal. 17, 2957-2968. 